\title{
Cocaine-Evoked Synaptic Plasticity of Excitatory Transmission in the Ventral Tegmental Area
}

\author{
Christian Lüscher \\ Department of Basic Neurosciences, University of Geneva, Geneva, Switzerland and Department \\ of Clinical Neurosciences, Geneva University Hospital, Geneva, Switzerland \\ Correspondence: Christian.luscher@unige.ch
}

Cocaine leads to a strong euphoria, which is at the origin of its recreational use. Past the acute effects, the drug leaves traces in the brain that persist long after it has been cleared from the body. These traces eventually shape behavior such that drug use may become compulsive and addiction develops. Here we discuss cocaine-evoked synaptic plasticity of glutamatergic transmission onto dopamine (DA) neurons of the ventral tegmental area (VTA) as one of the earliest traces after a first injection of cocaine. We review the literature that has examined the induction requirements as well as the expression mechanism of this form of plasticity and ask the question about its functional significance.

\begin{abstract}
A ddiction is a disease that starts with recreaAtional drug consumption but evolves to compulsive use in some people (Hyman 2005). Relapse can occur even after prolonged periods of abstinence, long after the substance has been eliminated from the body. This clinical reality points to the existence of persistent traces left by the addictive drug in the brain. Over the last decade drug-evoked synaptic plasticity has emerged as one of the cellular correlates of these drug traces (Lüscher and Malenka 2011). Although much research has now characterized drug-evoked plasticity at various synapses throughout the brain, the changes observed on excitatory afferents onto dopamine neurons in the VTA are of particular interest because they are among the earliest adaptations
\end{abstract}

that can be observed after a first drug exposure (Ungless et al. 2001). A single injection of cocaine to a mouse or a rat will affect excitatory transmission for days and although one dose of cocaine is not sufficient to get the rodent "addicted" (Kasanetz et al. 2010), these initial traces are likely to represent building blocks toward more permanent alterations in response to repetitive exposure. In support of this idea, all addictive drugs tested so far, but not other psychoactive substances such as fluoxetine or carbamazepine, trigger common synaptic adaptations in the VTA. A single dose of morphine, benzodiazepines, ethanol, amphetamines, or nicotine leads to the same synaptic changes as a single dose of cocaine (Saal et al. 2003; Brown et al. 2010; Mao et al. 2011). Therefore, studying

Editors: R. Christopher Pierce and Paul J. Kenny

Additional Perspectives on Addiction available at www.perspectivesinmedicine.org

Copyright (C) 2013 Cold Spring Harbor Laboratory Press; all rights reserved; doi: 10.1101/cshperspect.a012013

Cite this article as Cold Spring Harb Perspect Med 2013;3:a012013 


\section{Lüscher}

the molecular mechanisms of these early drugevoked traces in the VTA may lead to a better understanding of how addictive behavior is established.

\section{INDUCTION MECHANISM: MESOLIMBIC DA}

In a seminal study published about a decade ago (Ungless et al. 2001), it was observed that the ratio between the amplitude of $\alpha$-amino-3-hydroxy-5-methyl-4-isoxazolepropionic acid receptor (AMPAR)- and N-methyl D-aspartate receptor (NMDAR)-mediated EPSCs onto DA neurons in the VTA was increased by a systemic injection of a single dose of cocaine given $24 \mathrm{~h}$ before the preparation of the slices and the recording. The ex vivo AMPA/NMDA ratio was used (rather than measuring absolute amplitudes) because in the acute slice preparation the number of synapses recruited by the extracellular stimulation electrode cannot be controlled. Regardless of the mechanistic interpretation (see below), the increase of the AMPA/ NMDA ratio at glutamatergic synapses can be considered as a bona fide trace left by drug exposure. After a single injection to a mouse or a rat, the increase of the AMPA/NMDA ratio persists for $5 \mathrm{~d}$ but is reversed after $10 \mathrm{~d}$ (Ungless et al. 2001; Borgland et al. 2004; Kauer and Malenka 2007). In contrast, when an animal is trained to self-inject cocaine (which obviously results in many repetitive doses) the AMPA/ NMDA ratio remains increased for more than a month (Chen et al. 2008). Although not strictly comparable (it takes rats several days to learn to self-administer) these experiments suggest that the context of the injection may influence its persistence.

Because all addictive drugs cause an increase in the AMPA/NMDA ratio of DA neurons in the VTA, is there a common effect of these substances that drives the induction of this plasticity? It is well established that all addictive drugs strongly increase mesolimbic DA concentrations (Nestler 2005; Lüscher and Ungless 2006) (Fig. 1). Psychostimulants, for example, interfere with the reuptake of DA in target re- gions as well as in the VTA itself, where the DA neurons are capable of dendritic release (Sulzer 2011). Nicotine can directly depolarize DA neurons (Maskos et al. 2005), whereas opioids, benzodiazepines (Tan et al. 2010), cannabinoids (Melis 2004), and $\gamma$-hydroxybutyrate (GHB) (Cruz et al. 2004) preferentially inhibit GABA interneurons, thus causing a disinhibition. Through three distinct cellular mechanisms (interference with reuptake, direct excitation, and disinhibition of DA neurons) addictive drugs therefore converge to cause an increase of mesolimbic DA (Lüscher and Ungless 2006). Direct evidence that such increased mesolimbic dopamine is sufficient to cause a synaptic trace is provided by the experiments in which DA neurons are driven with optogenetic effectors. Such in vivo stimulation mimics drug-evoked synaptic plasticity (Brown et al. 2010).

The induction process is confined to the VTA because it can be reproduced in the slice preparation (Schilstrom 2006) and because a local infusion of the D1 (dopamine receptor) antagonist SCH23390 is sufficient to block the plasticity in response to optogenetic stimulation (Brown et al. 2010). This is in line with earlier reports that a systemic D1 antagonist blocks cocaine-evoked plasticity (Ungless et al. 2001). An additional induction requirement is the activation of NMDA receptors on the DA neurons. In inducible, conditional GluN1-KO mice in which NMDARs are selectively abolished in DA neurons, cocaine fails to elicit the synaptic trace (Engblom et al. 2008). The locus of the $\mathrm{D} 1 / 5 \mathrm{R}$ required for the induction is not established and may be a D1R expressed on glutamatergic afferents or alternatively a D5R found on the DA neurons themselves (Khan et al. 2000). This D1 or D5 receptor activation would prime NMDAR function (Schilstrom 2006; Argilli et al. 2008).

Another interesting observation is that orexin or corticotropin-releasing factor (CRF) antagonists abolish the appearance of cocaineevoked synaptic plasticity in the VTA (Bonci and Borgland 2008). Orexin neurons are located in the hypothalamus and project among several targets to the VTA, where they make synapses onto DA neurons, particularly in the caudiomedial 


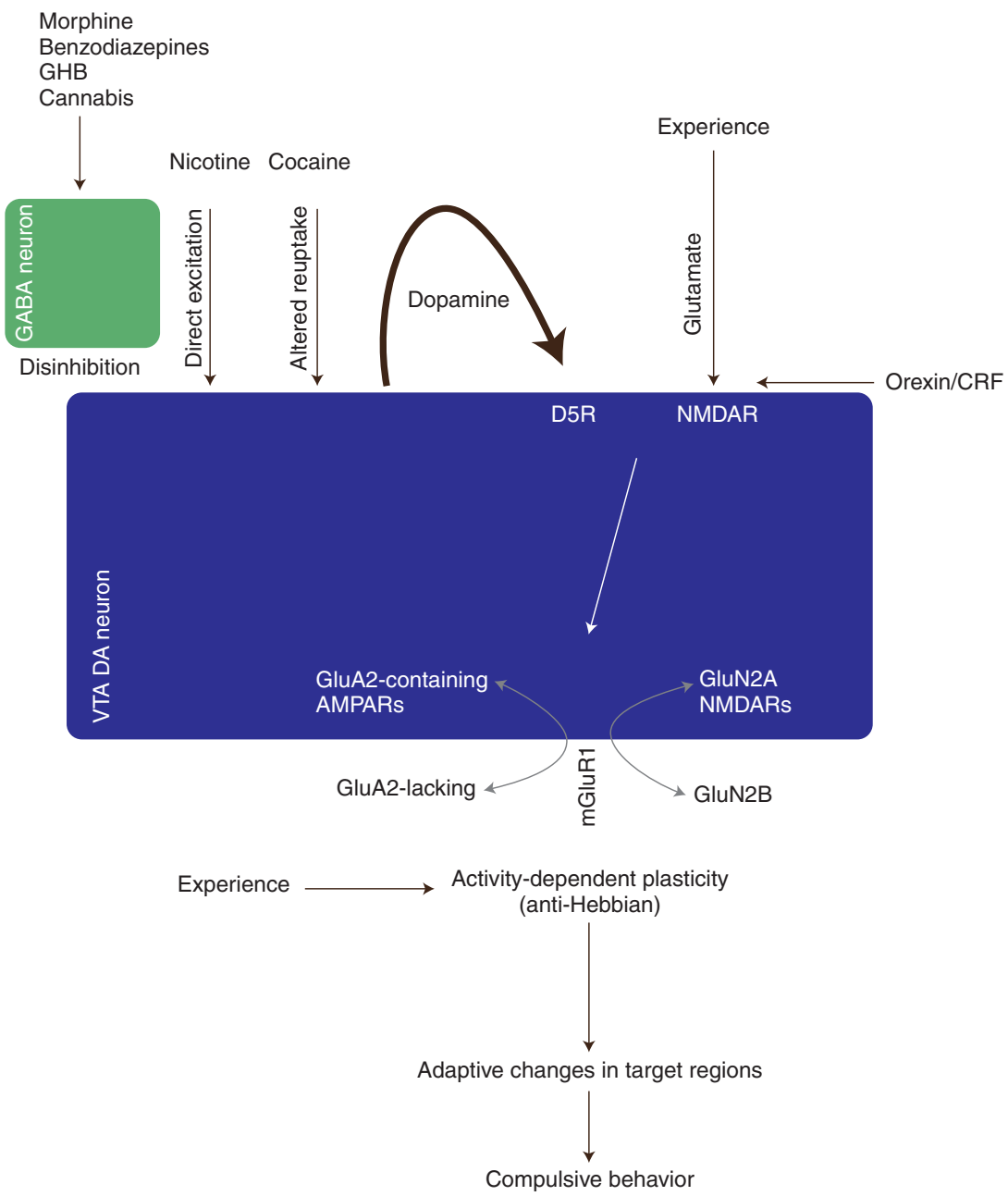

Figure 1. Schematic of the induction and expression mechanism of cocaine-evoked synaptic plasticity in dopamine neurons of the VTA. Addictive substances use distinct cellular mechanisms to cause a surge in dopamine, which through D1-like receptors, in conjunction with NMDAR, cause a redistribution of AMPAR and NMDARs. As a consequence the rules for activity-dependent plasticity are altered, which through yet to be identified signaling pathways leads to adaptive changes in target regions of the VTA. Once such adaptive changes are consolidated, compulsive behavior becomes apparent. For details, see text.

region (Vittoz et al. 2008). According to these studies, the orexin-1 receptor expressed on the surface of the DA neurons drives (via PKC) an increase in NMDAR function, a prerequisite for the long-lasting cocaine-evoked plasticity described above. In addition, a study monitoring c-fos as a marker of neuronal activity suggests that there is indeed an increased activity of orexin afferents during drug-related behavior (AstonJones et al. 2010). Taken together these pieces of data suggest that orexin plays a role as a gatekeeper of the cocaine-evoked effects on glutamatergic transmission. However, more studies with genetic manipulations and selective control of the activity of the orexin projections will be needed to achieve a comprehensive understanding.

CRF also potentiates NMDARs (via CRF2 receptors and similar to orexin by way of protein kinase $\mathrm{C}[\mathrm{PKC}]$ ) that again prime the synapse for the effects of cocaine (Ungless et al. 2003). 


\section{Lüscher}

However, the presence of a large Ih current, which was used to identify DA neurons in this study (Ungless et al. 2003), has subsequently been shown not to apply to all DA neurons (Lammel et al. 2008; Brown et al. 2009). Future studies will have to determine whether this effect applies to all DA neurons of the VTA. In addition it will have to be established from where the CRF afferents arrive and under which circumstances they are activated. Although orexin's and CRF's effect on the VTA share many commonalities, it also remains to be shown whether the changes of the NMDAR function are mechanistically identical.

The signaling pathway of cocaine-evoked synaptic plasticity relies on PKC activation, but is only incompletely known. A recent study also implicated PKM $\zeta$ (Ho et al. 2011), based on pharmacological interventions that abolished the appearance of cocaine-evoked plasticity and subsequent activity-dependent potentiation.

\section{EXPRESSION MECHANISM}

The increase of the AMPA/NMDA ratio is not the only change observed at excitatory inputs onto VTA DA neurons in response to addictive drugs. The current-voltage curve of AMPAREPSCs is also affected. Although linear at baseline or after saline control injections, a change in slope is observed after a single injection of cocaine (Bellone and Lüscher 2006; Argilli et al. 2008) and other additive drugs (Heikkinen et al. 2009; Wiltgen et al. 2010), indicating that AMPAR-EPSCs in drug-treated animals are inwardly rectifying.

Inward rectification is a property conferred by endogenous polyamines that inhibit AMPARs at positive potentials (Nishida and MacKinnon 2002). Rectification can be quantified by calculating the ratio of the chord conductance measured at negative over positive potentials. After drug treatment this rectification index typically has a value of 2 compared to a rectification index of 1 in cells from naïve or saline-injected mice. Such partial rectification suggests that the drug treatment causes the insertion of AMPARs that lack the subunit GluA2 into synapses of DA neurons (Bellone and Lü- scher 2006; Mameli et al. 2007). Most AMPARs in the central nervous system (CNS) contain at least one GluA2 subunit, which through posttranslational editing has an arginine residue in the pore region. This arginine modification regulates the single channel conductance for monovalent ions such as $\mathrm{Na}^{+}$and $\mathrm{K}^{+}$, makes the channel impermeable for $\mathrm{Ca}^{2+}$, and blocks the polyamine binding site (Seeburg 2003). Because this editing mechanism is also crucial for masking an ER retention signal, only edited GluA2-containing receptors reach the cell surface. This is in contrast with GluA1 homomeric or GluA1/3 heteromeric channels, which are made of subunits that are not subject to editing. The crucial residues in the pore regions of these subunits are glutamine. As a consequence, GluA1 homomeric or GluA1/3 heteromeric channels have a larger single channel conductance for sodium, are calcium permeable, and are inhibited by polyamines at positive potentials. Data from expression systems (reviewed in Liu and Zukin 2007) and the analysis of the constitutive knockout mice in DA neurons (Engblom et al. 2008) indicate that pure GluA2-lacking receptors are fully rectifying (i.e., no current flows at positive potentials). In native receptors, this may not always be the case as rectification is also determined by auxiliary subunits, such as the Tarps (Kelly et al. 2009).

Regardless of the exact molecular mechanism, the appearance of rectifying AMPAREPSCs after drug exposure suggests that some synapses contain a fraction of AMPARs, which lack the GluA2 subunit. In line with this interpretation, drug treatment significantly increases the mean single channel conductance of AMPARs (Mameli et al. 2007). These changes occur without affecting receptor number, however, as revealed by statistical analysis of the trial-to-trial fluctuation of EPSC amplitude (nonstationary fluctuation analysis). This model is further confirmed by electron microscopy, in which immunogold labeling shows a significant redistribution of the GluA2 subunit. After cocaine treatment, its cytoplasmic pool strongly grows at the expense of the pool of receptors at the surface (Mameli et al. 2007; Brown et al. 2010). 
Synaptic Plasticity of Excitatory Transmission

But how can the AMPA/NMDA ratio increase (particularly because both components are typically measured at $+40 \mathrm{mV}$ ) if the inserted AMPARs do not conduct current at positive potentials? Initially, studies suggested that, in contrast to the AMPARs, the NMDA component remained unchanged (Ungless et al. 2001). This was based on the observation that bath application of NMDA elicited currents of similar magnitude in slices of mice regardless of whether they were exposed to cocaine or saline. However, this approach has the disadvantage of activating synaptic as well as extrasynaptic receptors. A direct quantification of the amplitude of NMDA-EPSCs using standard extracellular stimulation techniques also remains problematic because across different slices the number of synapses stimulated (a parameter that crucially determines the amplitude) cannot be controlled. A technique that circumvents these problems is to elicit unitary responses at single synapses by generating a small artificial source of glutamate. This can be achieved using a two-photon laser to photolyze-caged glutamate in proximity of a synapse. Although this technique is well established for several brain regions (Bloodgood et al. 2009) and typically relies on the presence of a dendritic spine to identify the locus of the synapse, its validation is more difficult in the aspiny synapses of DA neurons of the VTA. Nevertheless, an iterative placement of the laser focus while optimizing kinetics and light power can reveal "hot spots" that fulfill the criteria of unitary EPSCs and allows one to compare absolute EPSC amplitudes in slices from saline versus cocaine-treated animals (Mameli et al. 2011). This approach not only confirmed the existence of rectifying AMPAR-EPSCs but also showed that NMDAREPSCs are significantly smaller after the drug treatment. These smaller NMDA currents may occur from a redistribution of NMDAR subunit composition (Schilstrom 2006; Bellone et al. 2012).

In summary, increase of the AMPA/NMDA ratio after drug exposure is owing to both a decrease of the NMDAR component and an increase in AMPA-EPSCs because of insertion of GluA2-lacking AMPARs.

\section{CONSEQUENCES FOR SYNAPTIC FUNCTION}

Although several elements of the induction and expression mechanisms are well described, we know very little about the consequences of drug-evoked synaptic plasticity in the VTA. How does the redistribution of glutamate receptors affect synaptic transmission and activity-dependent plasticity? Is the drug-evoked plasticity encoding specific elements of the drug-associated experience? Or is it rather a form of metaplasticity permissive for additional adaptive changes?

A first element that still requires clarification is the identification of the actual anatomical projection that is affected. One thing is clear: the early form of cocaine-evoked synaptic plasticity in DA neurons is limited to the cells in the VTA because neighboring DA neurons of the substantia nigra or GABA neurons in the VTA remain unaffected by a single injection (Ungless et al. 2001). The selectivity for VTA DA neurons along with the requirement for the activation of NMDARs expressed on these cells suggest that activity of afferent glutamatergic projections during the drug exposure is crucial. Although VTA DA neurons receive converging inputs from many upstream nuclei (e.g., prefrontal cortex [PFC], brain stem, amygdala), it is likely that not all undergo drug-evoked synaptic plasticity. In fact, when the plasticity is probed with single synapse resolution there are synapses that remain unaffected (Mameli et al. 2011). However, thanks to the advent of systematic projection targeting with optogenetic effectors, the identification of the relevant afferents should only be a matter of time.

The idea that a portion of DA neurons are part of a specific reward circuit that can undergo drug-evoked changes is shown by the observation that DA cells projecting to the nucleus accumbens (NAc), but not those that project to the PFC, express drug-evoked plasticity. Indeed, recent work selecting the neurons based on the presence of a fluorescent marker that was previously injected in various projection areas (so-called retrobeads) has found that mesocortical DA neurons exhibit an increase in the 
C. Lüscher

AMPAR/NMDAR ratio following an injection of formalin to the hindpaw (Lammel et al. 2011). Whether this observation can be generalized to any salient but aversive stimulus will require additional work. In particular, it will have to be investigated whether these experiments using a painful stimulus engage a similar mechanism as stress, which also leads to an increase in AMPAR/NMDAR ratio (e.g., forced swim test) (Saal et al. 2003).

Regardless of these questions, it is clear that DA neurons do not constitute a homogenous population. An electrical footshock inhibits most DA neurons (and excites a few), presumably indirectly after activation of upstream GABA neurons ( $T a n$ et al. 2012; van Zessen et al. 2012). However, when monitoring firing properties of DA neurons in vivo, it was observed that a small subpopulation, typically located in the lateral parts of the VTA, is excited by aversive stimuli (Brischoux et al. 2009; Bromberg-Martin et al. 2010). If such excitation is also driven in part by excitatory inputs that activate NMDARs, then this may help to understand the differential induction criteria observed in classes of DA neurons defined by their projection target (Lammel et al. 2011).

Another line of research, which may help to understand the functional consequences of drug-evoked plasticity in the VTA is the observation that the changes induced by cocaine in adult mice bring about many features of an immature synapse (Bellone et al. 2011). A systematic characterization of excitatory afferents onto DA neurons of the VTA for the first month revealed that AMPA-EPSCs are rectifying during the first week of life and linear by the third week. In parallel, the amplitudes of NMDA-EPSCs grow larger during the same period (as can be inferred by the change in AMPA/NMDA ratio). The NMDAR-EPSC changes are in part owing to a switch in subunit composition; although GluN2B is the predominate partner of the obligatory subunit GluN1 in the early neonatal period, GluN2A makes up the majority of receptors in adolescent and adult mice. Interestingly, neonatal NMDA receptors flux very little calcium. This may have something to do with their subunit composition, which in addition to GluN1 and GluN2B, may also contain GluN3, known to confer a very low calcium conductance (Paoletti 2011). Regardless of the precise molecular mechanism, the fact is, the major source for synaptic calcium in neonatal synapses is GluA2-lacking AMPARs, whereas this switches to an NMDARdependent calcium source in adult mice.

Given this developmental profile, the idea has been put forward that cocaine exposure in adult mice can reopen a critical period similar to that during development and thus change the rules for the induction of subsequent activity-dependent synaptic plasticity (Mameli et al. 2011). In naïve animals coordinated activity involving glutamate release onto depolarized NMDARs leads to efficient calcium influx and synaptic potentiation. After cocaine treatment this protocol is no longer efficient, presumably because of the reduced calcium permeability of NMDARs. However, glutamate release onto a hyperpolarized cell now becomes an efficient induction protocol because of the occurrence of calcium-permeable AMPARs. The consequences on the network function have not been worked out in detail. In any case these observations strongly suggest that this early form of drugevoked synaptic plasticity is actually a metaplasticity permissive for further adaptive changes with time and repetitive drug exposure. In line with this idea, it was observed that the changes of excitatory transmission onto medium spiny neurons of the NAc that are typically observed after several injections (of cocaine?) can be prevented if the GluA2-lacking AMPARs are quickly removed in the VTA DA neurons after each injection (Mameli et al. 2010; see also below). Conversely synaptic adaptations in the NAc are observed more than a month after a single injection of cocaine, provided that the adaptive changes persist in the VTA.

\section{REVERSING DRUG-EVOKED SYNAPTIC PLASTICITY}

After a single passive injection of cocaine, calcium-permeable AMPARs and reduced NMDAREPSCs can be observed for about $5 \mathrm{~d}$ (Ungless et al. 2001; Borgland et al. 2004; Bellone and Lüscher 2006). The persistence is prolonged if 
Synaptic Plasticity of Excitatory Transmission

the injection is self-controlled. For example, after 2 wk of self-administration of cocaine, the synaptic changes persist for several months (Chen et al. 2008). In all cases synaptic transmission eventually returns to baseline, which requires the activation of mGluR1 receptors (Bellone and Lüscher 2006). The involvement of mGluR1 is shown by the observation that pharmacological enhancement of mGluR1 causes a reversal within minutes, whereas conversely the disruption of the mGluR1 interaction with Homer1C makes the synaptic plasticity persistent (Mameli et al. 2010). Moreover, constitutive mGluR1 KO mice have rectifying AMPAREPSCs throughout their lives (Bellone et al.
2011). Besides the VTA, studies from both the amygdala (Clem and Huganir 2010) and the NAc (McCutcheon et al. 2011) provide evidence that mGluR1-LTD serves as a general mechanism (Lüscher and Huber 2010) by which GluA2lacking AMPARs are removed from the synapse.

The mGluR1-induced reversal of cocaineevoked synaptic plasticity (sometimes also termed mGluR1-LTD) depends on protein synthesis. In fact, mGluR1 through mTOR signaling triggers the translation of the GluA2 subunit, most likely from preexisting mRNA (Fig. 2) (Mameli et al. 2007). This conclusion was reached by RNA interference, simply by including the siRNA against GluA2 into the patch pipette. This shuts
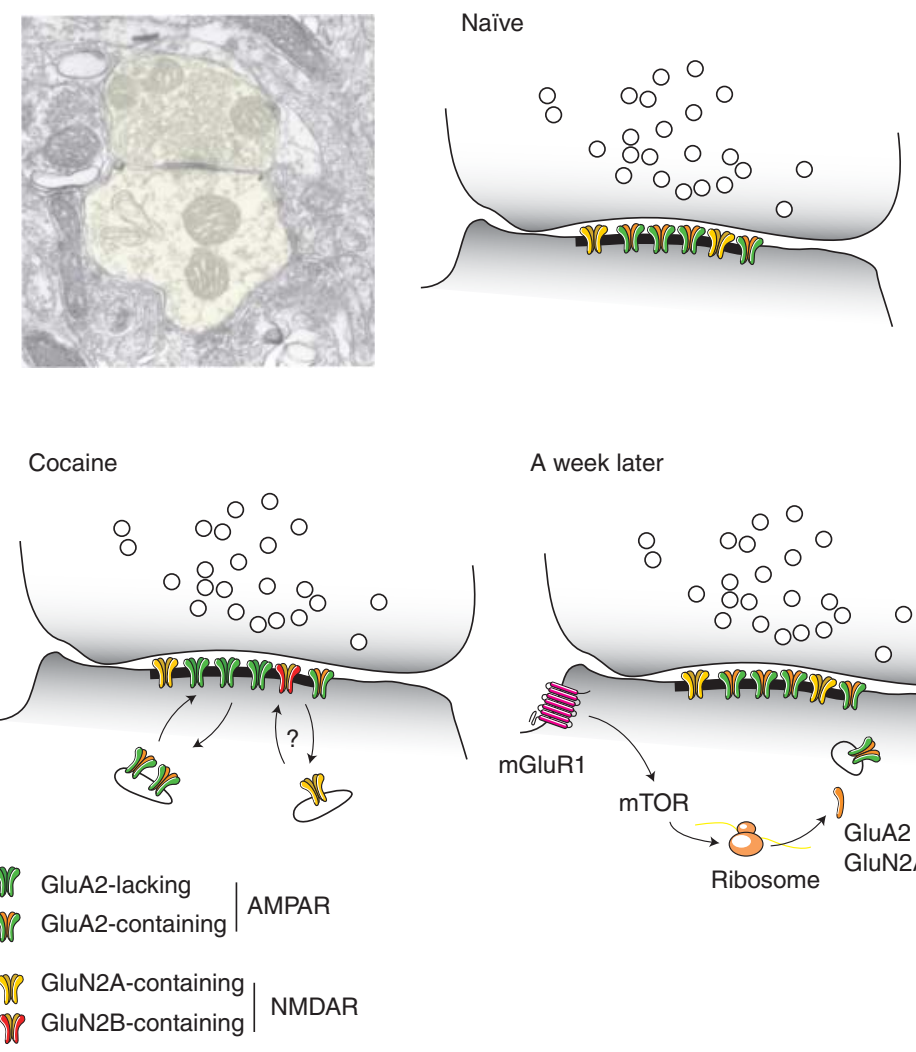

A week later

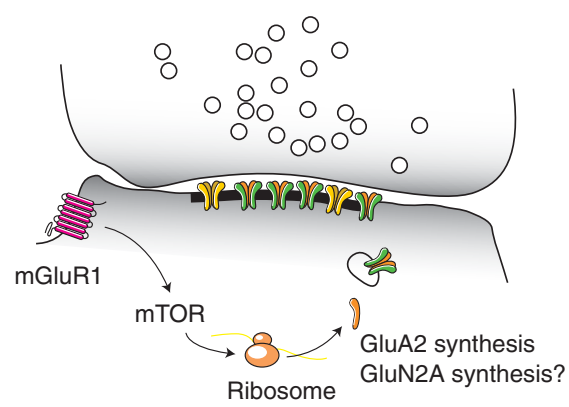

Figure 2. Receptor subunit redistribution and reversal of cocaine-evoked synaptic plasticity in DA neurons. Synapses between glutamatergic afferents and dendrites of VTA dopamine neurons are aspiny. (Top left panel, Electron microscopy picture, courtesy of Rafael Lujan.) Baseline transmission is mediated by GluA2-containing AMPARs and GluN2A-dominated NMDARs. After cocaine exposure GluA2-lacking AMPARs are inserted and NMDARs have a high content of GluN2B (actual exchange of receptors likely, but has not been directly shown). With strong activity, perisynaptic mGluR1s are activated, which through mTOR synthetize new GluA2 subunits. Whether NMDARs are also affected by mGluR1 signaling remains to be investigated. 


\section{Lüscher}

down the synthesis machinery within minutes and thus prevents de novo synthesis, an approach that has also been successfully used to show the involvement of Arc in other forms of protein-synthesis-dependent synaptic plasticity (Mameli et al. 2007; Lüscher and Huber 2010).

\section{BEHAVIORAL REPERCUSSIONS OF DRUG-EVOKED PLASTICITY IN THE VTA}

Linking cocaine-evoked synaptic plasticity in DA neurons of the VTA to a specific behavior has been difficult and the initial attempts looking into various forms of drug-adaptive behavior were not successful. For example, self-administration, behavioral sensitization, and unbiased conditioned place preference, all behaviors that occur with a time course similar to the time course of drugevoked synaptic plasticity in the VTA, remained unaffected when plasticity was inhibited (e.g., conditional, inducible GluN1 KO) or quickly reversed (mGluR1 positive modulator) (Engblom et al. 2008; Zweifel et al. 2008). It was only when much later forms of adaptive behavior such as drug-triggered reinstatement or cue-induced cocaine seeking were examined that there was a significant effect of inhibiting plasticity. This was shown by a reduction of these behaviors in mice that lack NMDARs in VTA DA neurons and thus do not show cocaine-evoked synaptic plasticity. This is very surprising because these behaviors can onlybe elicited by several injections of cocaine and develop with a delayed time course compared to the plasticity in the VTA, which can be observed within hours after drug injection. Although many details remain to be investigated, the most likely explanation is that the changes in the VTA are merely permissive for additional adaptations at other synapses, for example in the NAc, which then eventually will have a behavioral impact. In line with this interpretation, direct pharmacological or optogenetic manipulations of plasticity in the NAc have a more direct impact on behavior.

\section{CONCLUSIONS}

Drug-evoked synaptic plasticity is one of the earliest traces that becomes apparent soon after addictive drugs have been eliminated from the body. It is induced by excessive DA release within the VTA and by activity of glutamatergic synapses sufficient to activate NMDARs on the DA neurons. The expression mechanism involves a coordinated exchange of both AMPARs as well as NMDARs. In the case of AMPARs, the initially GluA2-containing receptors are exchanged for GluA2-lacking ones. A similar redistribution of NMDARs made of different subunits is also possible, but needs experimental confirmation. As a consequence excitatory transmission onto DA neurons is potentiated (GluA2-lacking AMPARs have a high single channel conductance) and the requirements for efficient synaptic calcium entry altered. Hyperpolarizing the postsynaptic neurons now facilitates calcium flux and can lead to further activity-dependent potentiation by an anti-Hebbian mechanism (Mameli et al. 2011). Such a metaplasticity has a permissive role for adaptive mechanisms engaged by subsequent drug exposure, gradually remodeling the circuitry and eventually changing behavior toward compulsive drug use without control (i.e., addiction).

\section{REFERENCES}

Argilli E, Sibley DR, Malenka RC, England PM, Bonci A. 2008. Mechanism and time course of cocaine-induced long-term potentiation in the ventral tegmental area. $J$ Neurosci 28: 9092-9100.

Aston-Jones G, Smith RJ, Sartor GC, Moorman DE, Massi L, Tahsili-Fahadan P, Richardson KA. 2010. Lateral hypothalamic orexin/hypocretin neurons: A role in reward-seeking and addiction. Brain Res 1314: 74-90.

Bellone C, Lüscher C. 2006. Cocaine triggered AMPA receptor redistribution is reversed in vivo by mGluR-dependent long-term depression. Nat Neurosci 9: 636-641.

Bellone C, Mameli M, Lüscher C. 2011. In utero exposure to cocaine delays postnatal synaptic maturation of glutamatergic transmission in the VTA. Nat Neurosci 1-9.

Bellone C, Yuan T, Mameli M, Lüscher C. 2012. Cocaine reopens a critical period of postnatal synaptic development: NMDA receptor plasticity at VTA synapses. FENS (Abstr 75.1).

Bloodgood BL, Giessel AJ, Sabatini BL. 2009. Biphasic synaptic Ca influx arising from compartmentalized electrical signals in dendritic spines. PLoS Biol 7: e1000190.

Bonci A, Borgland S. 2008. Role of orexin/hypocretin and CRF in the formation of drug-dependent synaptic plasticity in the mesolimbic system. Neuropharmacology 56 (Suppl 1): 107-111. 
Borgland SL, Malenka RC, Bonci A. 2004. Acute and chronic cocaine-induced potentiation of synaptic strength in the ventral tegmental area: Electrophysiological and behavioral correlates in individual rats. J Neurosci 24: 7482-7490.

Brischoux F, Chakraborty S, Brierley DI, Ungless MA. 2009. Phasic excitation of dopamine neurons in ventral VTA by noxious stimuli. Proc Natl Acad Sci 106: 4894-4899.

Bromberg-Martin ES, Matsumoto M, Hikosaka O. 2010. Dopamine in motivational control: Rewarding, aversive, and alerting. Neuron 68: 815-834.

Brown MTC, Henny P, Bolam JP, Magill PJ. 2009. Activity of neurochemically heterogeneous dopaminergic neurons in the substantia nigra during spontaneous and driven changes in brain state. J Neurosci 29: 2915-2925.

Brown MTC, Bellone C, Mameli M, Labouèbe G, Bocklisch C, Balland B, Dahan L, Luján R, Deisseroth K, Lüscher C. 2010. Drug-driven AMPA receptor redistribution mimicked by selective dopamine neuron stimulation. PLoS ONE 5: e15870.

Chen BT, Bowers MS, Martin M, Hopf FW, Guillory AM, Carelli RM, Chou JK, Bonci A. 2008. Cocaine but not natural reward self-administration nor passive cocaine infusion produces persistent LTP in the VTA. Neuron 59: $288-297$.

Clem RL, Huganir RL. 2010. Calcium-permeable AMPA receptor dynamics mediate fear memory erasure. Science 330: $1108-1112$

Cruz HG, Ivanova T, Lunn M-L, Stoffel M, Slesinger PA, Lüscher C. 2004. Bi-directional effects of GABAB receptor agonists on the mesolimbic dopamine system. Nat Neurosci 7: 153-159.

Engblom D, Bilbao A, Sanchis-Segura C, Dahan L, PerreauLenz S, Balland B, Parkitna JR, Luján R, Halbout B, Mameli M, et al. 2008. Glutamate receptors on dopamine neurons control the persistence of cocaine seeking. Neuron 59: 497-508.

Heikkinen AE, Möykkynen TP, Korpi ER. 2009. Long-lasting modulation of glutamatergic transmission in VTA dopamine neurons after a single dose of benzodiazepine agonists. Neuropsychopharmacology 34: 290-298.

Ho S-Y, Chen C-H, Liu T-H, Chang H-F, Liou J-C. 2011. Protein kinase $M \zeta$ is necessary for cocaine-induced synaptic potentiation in the ventral tegmental area. Biol Psychiatry 71: 706-713.

Hyman S. 2005. Addiction: A disease of learning and memory. Am J Psychiatry 162: 1414.

Kasanetz F, Deroche-Gamonet V, Berson N, Balado E, Lafourcade M, Manzoni O, Piazza PV. 2010. Transition to addiction is associated with a persistent impairment in synaptic plasticity. Science 328: 1709-1712.

Kauer JA, Malenka RC. 2007. Synaptic plasticity and addiction. Nat Rev Neurosci 8: 844-858.

Kelly L, Farrant M, Cull-Candy SG. 2009. Synaptic mGluR activation drives plasticity of calcium-permeable AMPA receptors. Nat Neurosci 12: 593-601.

Khan ZU, Gutiérrez A, Martín R, Peñafiel A, Rivera A, la Calle de A. 2000. Dopamine D5 receptors of rat and human brain. Neuroscience 100: 689-699.

Lammel S, Hetzel A, Häckel O, Jones I, Liss B, Roeper J. 2008. Unique properties of mesoprefrontal neurons within a dual mesocorticolimbic dopamine system. Neuron 57: 760-773.

Lammel S, Ion DI, Roeper J, Malenka RC. 2011. Projectionspecific modulation of dopamine neuron synapses by aversive and rewarding stimuli. Neuron 70: 855-862.

Liu SJ, Zukin RS. 2007. $\mathrm{Ca}^{2+}$-permeable AMPA receptors in synaptic plasticity and neuronal death. Trends Neurosci 30: $126-134$.

Lüscher C, Huber KM. 2010. Group 1 mGluR-dependent synaptic long-term depression: Mechanisms and implications for circuitry and disease. Neuron 65: 445-459.

Lüscher C, Malenka RC. 2011. Drug-evoked synaptic plasticity in addiction: From molecular changes to circuit remodeling. Neuron 69: 650-663.

Lüscher C, Ungless MA. 2006. The mechanistic classification of addictive drugs. PLoS Med 3: e437.

Mameli M, Balland B, Lujan R, Luscher C. 2007. Rapid synthesis and synaptic insertion of GluR2 for mGluR-LTD in the ventral tegmental area. Science 317: 530-533.

Mameli M, Halbout B, Creton C, Engblom D, Parkitna JR, Spanagel R, Lüscher C. 2010. Cocaine-evoked synaptic plasticity: Persistence in the VTA triggers adaptations in the NAc. Nat Neurosci 12: 1036-1041.

Mameli M, Bellone C, Brown MTC, Lüscher C. 2011. Cocaine inverts rules for synaptic plasticity of glutamate transmission in the ventral tegmental area. Nat Neurosci 14: 414-416.

Mao D, Gallagher K, Mcgehee DS. 2011. Nicotine potentiation of excitatory inputs to ventral tegmental area dopamine neurons. J Neurosci 31: 6710-6720.

Maskos U, Molles BE, Pons S, Besson M, Guiard BP, Guilloux J-P, Evrard A, Cazala P, Cormier A, MameliEngvall M, et al. 2005. Nicotine reinforcement and cognition restored by targeted expression of nicotinic receptors. Nature 436: 103-107.

McCutcheon JE, Loweth JA, Ford KA, Marinelli M, Wolf ME, Tseng KY. 2011. Group I mGluR activation reverses cocaine-induced accumulation of calcium-permeable AMPA receptors in nucleus accumbens synapses via a protein kinase C-dependent mechanism. J Neurosci 31: $14536-14541$.

Melis M. 2004. Endocannabinoids mediate presynaptic inhibition of glutamatergic transmission in rat ventral tegmental area dopamine neurons through activation of CB1 receptors. J Neurosci 24: 53-62.

Nestler EJ. 2005. Is there a common molecular pathway for addiction? Nat Neurosci 8: 1445-1449.

Nishida M, MacKinnon R. 2002. Structural basis of inward rectification: Cytoplasmic pore of the $\mathrm{G}$ protein-gated inward rectifier GIRK1 at 1.8 A resolution. Cell 111: 957-965.

Paoletti P. 2011. Molecular basis of NMDA receptor functional diversity. Eur J Neurosci 33: 1351-1365.

Saal D, Dong Y, Bonci A, Malenka RC. 2003. Drugs of abuse and stress trigger a common synaptic adaptation in dopamine neurons. Neuron 37: 577-582.

Schilstrom B. 2006. Cocaine enhances NMDA receptor-mediated currents in ventral tegmental area cells via dopamine D5 receptor-dependent redistribution of NMDA receptors. J Neurosci 26: 8549-8558. 


\section{Lüscher}

Seeburg P. 2003. Regulation of ion channel/neurotransmitter receptor function by RNA editing. Curr Opin Neurobiol 13: 279-283.

Sulzer D. 2011. How addictive drugs disrupt presynaptic dopamine neurotransmission. Neuron 69: 628-649.

Tan KR, Brown M, Labouèbe G, Yvon C, Creton C, Fritschy J-M, Rudolph U, Lüscher C. 2010. Neural bases for addictive properties of benzodiazepines. Nature 463: 769-774.

Tan KR, Yvon C, Turiault M, Mirzabekov JJ, Doehner J, Labouèbe G, Deisseroth K, Tye KM, Lüscher C. 2012. GABA neurons of the VTA drive conditioned place aversion. Neuron 73: 1173-1183.

Ungless MA, Whistler JL, Malenka RC, Bonci A. 2001. Single cocaine exposure in vivo induces long-term potentiation in dopamine neurons. Nature 411: 583-587.

Ungless MA, Singh V, Crowder TL, Yaka R, Ron D, Bonci A. 2003. Corticotropin-releasing factor requires CRF binding protein to potentiate NMDA receptors via CRF receptor 2 in dopamine neurons. Neuron 39: 401407.

van Zessen R, Phillips JL, Budygin EA, Stuber GD. 2012. Activation of VTA GABA neurons disrupts reward consumption. Neuron 73: 1184-1194.

Vittoz NM, Schmeichel B, Berridge CW. 2008. Hypocretin /orexin preferentially activates caudomedial ventral tegmental area dopamine neurons. Eur J Neurosci 28: 1629 1640 .

Wiltgen BJ, Royle GA, Gray EE, Abdipranoto A, Thangthaeng $N$, Jacobs $N$, Saab F, Tonegawa S, Heinemann SF, O'Dell TJ, et al. 2010. A role for calcium-permeable AMPA receptors in synaptic plasticity and learning. PLoS ONE 5: e12818.

Zweifel L, Argilli E, Bonci A, Palmiter R. 2008. Role of NMDA receptors in dopamine neurons for plasticity and addictive behaviors. Neuron 59: 486-496. 


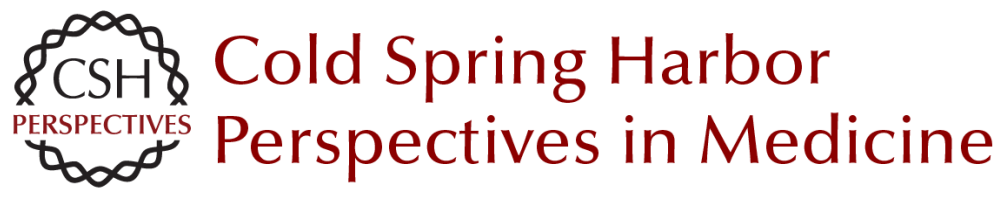

\title{
Cocaine-Evoked Synaptic Plasticity of Excitatory Transmission in the Ventral Tegmental Area
}

\author{
Christian Lüscher
}

Cold Spring Harb Perspect Med 2013; doi: 10.1101/cshperspect.a012013

\section{Subject Collection Addiction}

Developments from Bulk Optogenetics to

Single-Cell Strategies to Dissect the Neural

Circuits that Underlie Aberrant Motivational States

Jose Rodriguez-Romaguera, Vijay M.K.

Namboodiri, Marcus L. Basiri, et al.

Consequences of Parental Opioid Exposure on

Neurophysiology, Behavior, and Health in the

Next Generations

Fair M. Vassoler and Mathieu E. Wimmer

Animal Models of the Behavioral Symptoms of

Substance Use Disorders Louk J.M.J. Vanderschuren and Serge H. Ahmed

Translational Research in Nicotine Addiction Miranda L. Fisher, James R. Pauly, Brett Froeliger, et al.

Neonatal Opioid Withdrawal Syndrome (NOWS): A Transgenerational Echo of the Opioid Crisis Andrew E. Weller, Richard C. Crist, Benjamin C. Reiner, et al.

Impairment of Synaptic Plasticity by Cannabis, $\Delta^{\mathbf{9}}$ -THC, and Synthetic Cannabinoids

Alexander F. Hoffman, Eun-Kyung Hwang and Carl R. Lupica

Drug-Evoked Synaptic Plasticity of Excitatory

Transmission in the Ventral Tegmental Area Camilla Bellone, Michael Loureiro and Christian Lüscher

Opioid-Induced Molecular and Cellular Plasticity

of Ventral Tegmental Area Dopamine Neurons

Marie A. Doyle and Michelle S. Mazei-Robison
The Persistent Challenge of Developing Addiction

Pharmacotherapies

Sarah E. Swinford-Jackson, Charles P. O'Brien,

Paul J. Kenny, et al.

Opioid Modulation of the Gut-Brain Axis in Opioid-Associated Comorbidities

Li Zhang and Sabita Roy

Epigenetics of Drug Addiction

Andrew F. Stewart, Sasha L. Fulton and lan Maze

Genetic Vulnerability to Opioid Addiction

Brian Reed and Mary Jeanne Kreek

Glutamatergic Systems and Memory Mechanisms Underlying Opioid Addiction

Jasper A. Heinsbroek, Taco J. De Vries and Jamie Peters

Mechanisms of Nicotine Addiction

Marina R. Picciotto and Paul J. Kenny

Neural Substrates and Circuits of Drug Addiction Matthew W. Feltenstein, Ronald E. See and Rita A. Fuchs

The Role of the Central Amygdala in Alcohol

Dependence Marisa Roberto, Dean Kirson and Sophia Khom

For additional articles in this collection, see http://perspectivesinmedicine.cshlp.org/cgi/collection/ 